\title{
Conhecimentos e Atitudes Relativas a SIDA/AIDS em uma População de Favela do Rio de Janeiro
}

\author{
Knowledges and Attitudes Related to AIDS in \\ a Slum Population of Rio de Janeiro
}

\author{
João Claudio L. Fernandes' \\ Evandro da S. F. Coutinho ${ }^{2}$ \\ Alvaro Matida ${ }^{3}$
}

FERNANDES, J. C. L.; COUTINHO, E. da S. F. \& MATIDA, A. Knowledges and Attitudes

Related to AIDS in a Slum Population of Rio de Janeiro. Cad. Saude Públ., Rio de Janeiro, 8 (2): 176-182, abr/jun, 1992.

Aiming to evaluate the level of knowledge and attitudes related to AIDS, a survey was conducted in a sample of 13-45 year-old dwellers from Rocinha slum, Rio de Janeiro. The "Rapid Survey Methodology" was adopted mainly because of its low cost and the possibility to get fast answering to the most relevant questions.

Although most of the sample showed a good knowledge about the sexual transmission of HIV, over $50 \%$ referred to mosquito bite, blood donation and mouth kissing as ways of getting AIDS. In terms of condom use, only $57,6 \%$ of the respondents believe that this is a safe method for prevention, and $21,9 \%$ referred the eventual use of preservatives.

Keywords: AIDS; HIV Transmission; Knowledge; Behaviours; Prevention

\section{INTRODUÇÃO}

Desde que a Síndrome de Imunodeficiência Adquirida (SIDA/AIDS) foi registrada pela primeira vez no Brasil, em 1981, o estado do Rio de Janeiro vem ocupando o segundo lugar no total de casos da doença. Atualmente, $18 \%$ dos casos nacionais ocorrem nessa unidade da federação (SES,1990).

A maioria destes pacientes, no início da década de 80 , pertencia a grupos especificos, com fatores de risco bem definidos. No Rio de Janeiro, no periodo $1982-1984$, 68,8\% dos

\footnotetext{
' Pasto de Saúde da Associação dos Moradores e Amigos do Bairro Barcellos. Travessa Flores, 4, Favela da Rocinha, Rio de Janeiro, RJ, 22451-410. Brasil.

${ }^{2}$ Departamento de Epidemiologia e Métodos Quantitativos em Saúde da Escola Nacional de Saúde Pública. Rua Leopoldo Bulhöes, 1480, Manguinhos Rio de Janeiro, RJ, 21041-210, Brasil.

${ }^{3}$ Divisão de DST/AIDS, Secretaria de Estado de Saúde do Rio de Janeiro. Rua México 128, sala 410 , Rio de Janeiro, RJ, 20031-142, Brasil.
}

casos ocorreram entre indivíduos masculinos com prática homo/bissexual. Dessa época até o momento, além do aumento progressivo do número de casos, algumas mudanças importantes se observaram no padrão da epidemia (SES, 1990):

- redução proporcional de casos cujo fator de risco é a homo/bissexualidade masculina;

- redução de casos entre receptores de sangue e derivados;

- aumento proporcional de casos entre individuos com práticas heterossexuais;

- diminuição da razão homem:mulher de 9:1, em 1987, para 6:1, em 1989;

- aumento dos casos de SIDA/AIDS entre menores de 15 anos: taxa de crescimento de $61,8 \%$ entre 1986 e 1989 ;

- aumento de casos de transmissão materno-infantil entre doentes menores de 15 anos.

A análise dos dados de doença, que refletem o padrão de transmissão do vírus relativo ao final da década de 70 e ao longo dos anos 80 , 
e das taxas de infecção pelo vírus da Imunodeficiência Adquirida Humana (VIH), embora escassas e assistemáticas em nosso meio, permite identificar mudanças relevantes no perfil epidemiológico e na magnitude da SIDA/AIDS no estado do Rio de Janeiro. Em 1990, a soropositividade para o VIH-l entre doadores de sangue atingiu prevalências em torno de $0,35 \%$; neste mesmo ano, dados preliminares de um estudo mostram que $0,8 \%$ das puérperas/gestantes atendidas em instituições públicas da cidade do Rio de Janeiro são portadoras da infecção (May, 1991). Esses dados se revestem de uma dupla importância. Em primeiro lugar, traduzem um quadro mais recente da evolução da epidemia no estado em relação aos dados de doença. Em segundo, esses grupos pertencem, predominantemente, a estratos de baixo poder aquisitivo, onde o acesso à informação e a meios de prevenção é restrito. No caso da SIDA/AIDS, as estratégias para a redução das taxas de infecção disponiveis no momento se limitam ao controle de hemoderivados, à difusão de conhecimentos sobre a doença e seus mecanismos de transmissão, e à oferta de condições reais que possibilitem aos indivíduos mudanças de atitudes e comportamentos frente à transmissão do VIH.

Em função desse quadro e da necessidade de otimizar as ações do Programa Comunitário de Controle da AIDS, desenvolvido pela equipe do Posto de Saúde da Associação dos Moradores e Amigos do Bairro Barcellos (PS.AMABB), conduziu-se um estudo com o objetivo de avaliar o nivel de informação, percepção e comportamento frente à SIDA/AIDS em uma população de baixa renda.

\section{MATERIAL E MÉTODOS}

Para a realização deste estudo, adotou-se a metodologia denominada Rapid Survey Methodology (Frerichs \& Tar Tar, 1989), em virtude do seu baixo custo e por possibilitar respostas rápidas a questōes relativas ao nivel de conhecimento da comunidade, o que corresponde aos objetivos do trabalho e às caracteristicas de uma unidade de atenção primária à saúde.

\section{POPULAÇÃO DE REFERÊNCIA}

A favela da Rocinha possui cerca de $\mathbf{2 0 0 . 0 0 0}$ habitantes e situa-se no bairro de São Conrado, na zona sul da cidade do Rio de Janeiro. Segundo levantamento realizado pela equipe do Posto de Saúde, em 1986, sua populaçāo é composta, basicamente, por imigrantes nordestinos, oriundos principalmente do interior da Paraíba e Ceará. A atividade econômica predominante é a prestação de serviços, principalmente nos ramos de transportes, restaurantes, hotéis e serviços domésticos. A média de habitantes por domicilio é de 4,1 , e é comum a coabitação de até três gerações no mesmo quarto.

\section{AMOSTRA}

Optou-se pelo método simplificado de amostragem por conglomerado utilizado no Programa Ampliado de Imunizações (Henderson \& Sundaresan, 1982). Este método pressupõe uma seleção em dois estágios. No primeiro, são sorteados trinta setores (conglomerados) na área a ser estudada. Em seguida, seleciona-se, aleatoriamente, um ponto em cada conglomerado e uma direção a ser percorrida até que sejam encontradas sete pessoas que satisfaçam as condições para pertencer ao estudo. Desta forma, obteve-se uma amostra randômica de 210 pessoas entre 13 e 45 anos de idade, de ambos os sexos, que moravam nos domicílios sorteados.

\section{INSTRUMENTO}

$\mathrm{O}$ instrumento consistiu em um questionário com 32 perguntas com respostas fechadas, elaborado pela equipe do PS.AMABB, incorporando sugestões feitas pela Secretaria de Estado de Saúde - área programática AIDS e Associação Brasileira Interdisciplinar de AIDS (ABIA). Este questionário foi pré-testado em 30 pessoas, tendo sofrido algumas adaptações, sobretudo na linguagem utilizada.

\section{COLETA DE DADOS}

A equipe de entrevistadores foi composta por 
cinco moradores da comunidade que preenchiam os seguintes critérios: escolaridade mínima até a $4^{\mathrm{a}}$ série; idade entre 17 e 30 anos; bom desempenho durante o treinamento. Optou-se pela utilização de pessoas da própria comunidade, principalmente pelo conhecimento que possuem da região, facilitando a localização das áreas sorteadas. $O$ treinamento, com 10 horas de duração, consistiu de discussões sobre aspectos gerais da doença; aplicação do questionário entre os membros da equipe; aplicaçāo do questionário na comunidade, utilizando-se a estratégia do teste-reteste para avaliar o grau de concordância; e dramatização de situações passiveis de serem encontradas.

As entrevistas foram realizadas de 7 a 10 de setembro de 1990, aproveitando-se um fim de semana prolongado, para evitar distorções importantes na razão masculino/feminino. Deve-se ressaltar, a título de observação, o fato de terem sido identificados, na amostra, um caso de portador do vírus e dois parentes próximos de doentes VIH.

\section{ANÁLISE DOS DADOS}

Os dados coletados foram codificados e armazenados em microcomputador. $A$ análise dos dados consistiu na elaboração de tabelas de frequiência, tabelas de contingência, estratificações e medidas de associação (razão de produtos cruzados).

\section{RESULTADOS}

A população entrevistada consistiu, majoritariamente, de mulheres $(68,6 \%)$ e pessoas casadas $(65,7 \%)$, sendo a idade média dos entrevistados de 27,7 anos; $89,1 \%$ da amostra afirmaram saber ler e escrever.

Na Figura 1, observa-se que a principal fonte de informação sobre AIDS referida pelos entrevistados foram os meios de comunicação de massa, sobretudo a televisão $(69,1 \%)$, em contraste com a escola e serviços médicos, que, juntos, foram mencionados por apenas $4,3 \%$ dos entrevistados.

A Tabela 1 refere-se a perguntas gerais sobre o conhecimento da doença. Mais de $75 \%$ dos entrevistados responderam corretamente às perguntas relacionadas ao tratamento $\mathrm{e}$ ao estado de portador assintomático. No que se refere às formas de transmissão sexual, a proporção de respostas corretas superou $95 \%$ da amostra. Não foram encontradas diferenças estatisticamente significativas $(\mathrm{p}<0,05)$ quando os dados foram estratificados por sexo.

$\mathrm{Na}$ Tabela 2 encontram-se os dados referentes à transmissão não-sexual. Com relação ao uso de drogas injetáveis e transfusão de sangue, manteve-se um padrão elevado de respostas corretas. Verificou-se, entretanto, uma queda importante deste padrão quando foram investigadas as possibilidades de transmissão através da doação de sangue, beijo na boca e picada de insetos. Assim como na tabela anterior, não foram identificadas diferenças estatisticamente significativas entre homens e mulheres.

FIGURA 1 - Principais Fontes de Informação

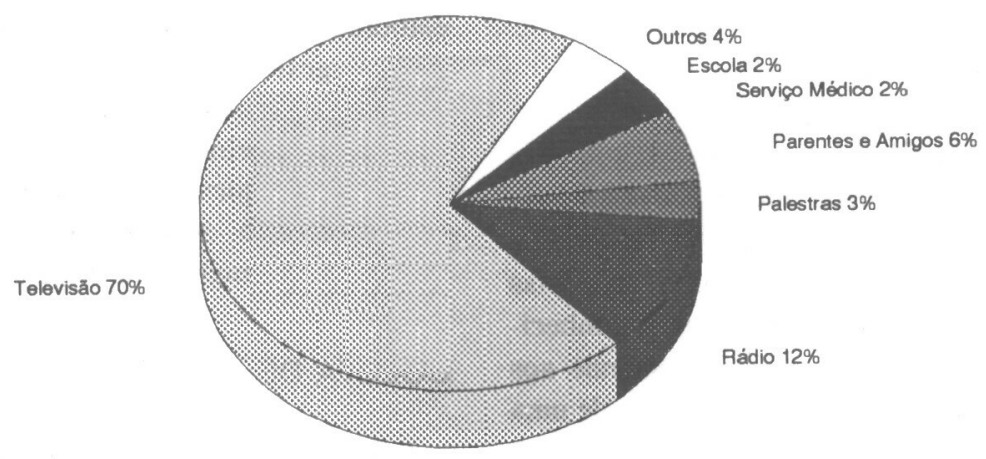


TABELA 1 - Conhecimentos Gerais Sobre a Doença

\begin{tabular}{|c|c|c|c|c|c|c|}
\hline & \multicolumn{2}{|c|}{ Sim } & \multicolumn{2}{|c|}{ Não } & \multicolumn{2}{|c|}{ Não Sabe } \\
\hline & $\mathrm{n}$ & $\%$ & $\mathrm{n}$ & $\%$ & $\mathrm{n}$ & $\mathscr{w}$ \\
\hline Existência de vacina & 37 & 17,6 & 158 & 75,2 & 15 & 7,1 \\
\hline Existência de remédio & 32 & 15,2 & 161 & 76,7 & 17 & 8,1 \\
\hline $\begin{array}{l}\text { Uma pessoa pode ter o vírus e } \\
\text { não ter a doença }\end{array}$ & 163 & 77,6 & 38 & 18,1 & 8 & 3,8 \\
\hline $\begin{array}{l}\text { Uma pessoa de boa aparência pode } \\
\text { ter o virus }\end{array}$ & 173 & 82,4 & 29 & 13,8 & 7 & 3,3 \\
\hline $\begin{array}{l}\text { O vírus só pode ser transmitido por } \\
\text { via sexual }\end{array}$ & 37 & 17,6 & 170 & 81,0 & 3 & 1,4 \\
\hline $\begin{array}{l}\text { Contaminação por relação entre } \\
\text { homem sadio e mulher infectada }\end{array}$ & 202 & 96,2 & 7 & 3,3 & 1 & 0,5 \\
\hline $\begin{array}{l}\text { Contaminação por relação entre } \\
\text { mulher sadia e homem infectado }\end{array}$ & 203 & 96,7 & 6 & 2,9 & 1 & 0,5 \\
\hline $\begin{array}{l}\text { Contaminação por relação entre } \\
\text { homem sadio e homem infectado }\end{array}$ & 201 & 95,7 & 5 & 2,4 & 4 & 1,9 \\
\hline
\end{tabular}

TABELA 2 - Conhecimentos Sobre a Transmissão Não-Sexual

\begin{tabular}{lcccccc}
\hline & \multicolumn{2}{c}{ Sim } & \multicolumn{2}{c}{ Não } & \multicolumn{2}{c}{ Não Sabe } \\
& n & \% & \multicolumn{1}{c}{ n } & \% & n & $\%$ \\
\cline { 2 - 7 } Drogas injetáveis & 196 & 93,3 & 8 & 3,8 & 6 & 2,9 \\
Transfusão de sangue & 193 & 91,9 & 11 & 5,2 & 6 & 2,9 \\
Doação de sangue & 128 & 61,0 & 73 & 34,8 & 9 & 4,3 \\
Beijo na boca & 57 & 27,1 & 140 & 66,7 & 13 & 6,2 \\
Picada de mosquito & 95 & 45,2 & 101 & 48,1 & 14 & 6,7 \\
\hline \hline
\end{tabular}

A Figura 2 mostra os dados relativos ao uso de preservativos como método de prevenção da doença. Observamos que apenas $57,6 \%$ dos entrevistados acreditam que o uso destes evita a transmissão sexual da doença.

Em contraposição ao nivel de conhecimento referido anteriormente, mais da metade dos entrevistados $(64,8 \%)$ afirmou não ter modificado seu comportamento sexual em função da epidemia de SIDA/AIDS. O uso de preservativos só foi mencionado por 46 pessoas $(21,9 \%)$, sendo que destas, apenas 17 o utilizavam regularmente. Observou-se uma associação entre a referência à mudança de comportamento e o uso de preservativo $(\mathrm{rpc}=6,69, \mathrm{p}<0,001)$ (Tabela 3).
FIGURA 2 - Credibilidade no Preservativo como Meio de Prevenção

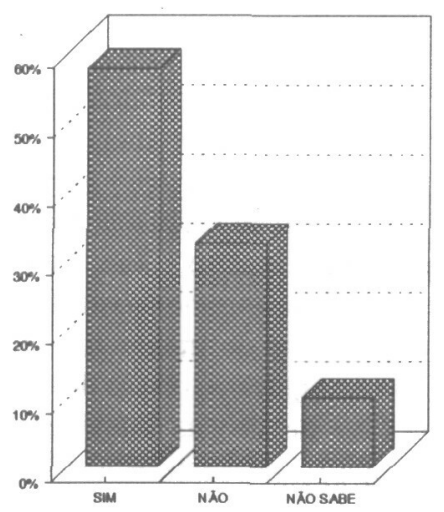


TABELA 3 - Referència a Mudança de Comportamento e Uso de Preservativo

\begin{tabular}{|c|c|c|c|c|c|c|c|c|}
\hline \multirow{3}{*}{$\begin{array}{c}\text { Mudança } \\
\text { de } \\
\text { Comportamento }\end{array}$} & \multicolumn{6}{|c|}{ Uso de Preservativo } & \multirow{2}{*}{\multicolumn{2}{|c|}{ Total }} \\
\hline & \multicolumn{2}{|c|}{$\operatorname{Sim}$} & \multicolumn{2}{|c|}{ Não } & \multicolumn{2}{|c|}{ Não Responde } & & \\
\hline & $\mathrm{n}$ & $\%$ & $\mathrm{n}$ & $\%$ & n & $\%$ & $\mathrm{n}$ & $\%$ \\
\hline Sim & 31 & 43,7 & 40 & 56,3 & 0 & 0 & 71 & 100,0 \\
\hline Não & 14 & 10,3 & 121 & 89,0 & 1 & 0,7 & 136 & 100,0 \\
\hline Não Sabe & 0 & 0 & 1 & 100,0 & 0 & 0 & 1 & 100,0 \\
\hline Não Responde & 1 & 50,0 & 0 & 0 & 1 & 50,0 & 2 & 100,0 \\
\hline Total & 46 & 21,9 & 162 & 77,1 & 2 & 1,0 & 210 & 100,0 \\
\hline
\end{tabular}

TABELA 4 - Conhecimento de Casos de AIDS* e Uso de Preservativo

\begin{tabular}{l|cccccc|ccc}
\hline \hline & \multicolumn{3}{|c}{ Usa } & \multicolumn{2}{c|}{ Não Usa } & \multicolumn{2}{c|}{ Não } & \multicolumn{2}{|c}{} \\
& \multicolumn{2}{|c}{ Preservativo } & \multicolumn{2}{c}{ Preservativo } & \multicolumn{2}{c}{ Responde } & \multicolumn{2}{c}{ Total } \\
& $\mathrm{n}$ & $\%$ & $\mathrm{n}$ & $\%$ & $\mathrm{n}$ & $\%$ & $\mathrm{n}$ & $\%$ \\
\hline $\begin{array}{l}\text { Conhece Casos } \\
\text { de AIDS }\end{array}$ & 10 & 19,2 & 42 & 80,8 & 0 & 0 & 52 & 100,0 \\
$\begin{array}{l}\text { Não Conhece } \\
\text { Casos de AIDS }\end{array}$ & 34 & 23,0 & 112 & 75,7 & 2 & 1,4 & 48 & 100,0 \\
Não Sabe & 2 & 20,0 & 8 & 80,0 & 0 & 0 & 10 & 100,0 \\
\hline Total & 46 & 21,9 & 162 & 77,1 & 2 & 1,0 & 210 & 100,0 \\
\hline \hline
\end{tabular}

$\left({ }^{\star}\right)$ entre parentes, vizinhos, colegas de escola e trabalho

Cerca de 1/4 da amostra relatou ter conhecido casos de SIDA/AIDS nos locais que freqüenta. Embora o uso de preservativos seja menos freqüente neste grupo, tal associacão não foi estatisticamente significativa ao nivel de 0,05 (Tabela 4).

\section{COMENTÁRIOS}

A partir dos resultados obtidos neste estudo, ficou evidenciada a importância da televisão enquanto fonte de informação sobre a SIDA/ AIDS para a população estudada. Há um elevado nível de conhecimento sobre a transmissão sexual, o estado de portador assintomático e a ausência de métodos de tratamento ou prevenção específicos. Algumas formas de transmissão não-sexual (uso de drogas injetáveis e transfusão de sangue) foram também referidas pela maioria dos entrevistados.

Resultados semelhantes foram observados em pelo menos dois estudos. Parker (1990) cita uma pesquisa realizada pelo Gallup International, em 1987-88, no Brasil, na qual a transfusão de sangue e o compartilhamento de seringas foram apontados como meios de transmissão da SIDA/AIDS por $97 \%$ dos entrevistados. Naquele estudo, $88 \%$ referiram o contato sexual íntimo com uma pessoa do mesmo sexo e $84 \%$ mencionaram o contato sexual íntimo com uma pessoa do sexo oposto como formas de transmissão.

Em outra pesquisa realizada com usuários do metrô de São Paulo (Munhoz et al., 1989), observou-se que o veículo de transmissão mais conhecido era o sangue, enquanto a secreção vaginal era o menos conhecido.

Dois fatos importantes observados em nosso estudo merecem ser destacados. O primeiro refere-se ao conhecimento equivocado quanto a determinadas formas de transmissão, como a doação de sangue, o beijo na boca e a picada de mosquito. O segundo indica a inexistência 
de uma correlação entre o conhecimento e a adoção de medidas de proteção individual contra a infecção.

O estudo com usuários do metrô paulista (Munhoz et al., 1989) também encontrou $40 \%$ de indivíduos que acreditavam que $o$ ato de doar sangue representava um risco de contrair a SIDA/AIDS. Em segundo lugar, o desconhecimento referia-se à transmissão pela saliva e através da picada de mosquito. Com relação à mudança de comportamento, a mesma pesquisa encontrou relato de modificação de hábitos em $55 \%$ dos entrevistados, sendo que metade destes optaram pelo parceiro sexual único e um terço, pelo uso do preservativo. Já na pesquisa do Gallup International citada por Parker (Parker, 1990 ), apenas $14 \%$ dos entrevistados disseram ter mudado o comportamento em decorrência da SIDA/AIDS.

Esses achados, assim como os de nossa pesquisa, são bem menores do que aqueles verificados por Parker (s/d) em um grupo de homens homo e bissexuais do Rio de Janeiro, onde $96 \%$ dos entrevistados revelaram ter mudado o comportamento sexual. Oitenta e seis por cento disseram usar preservativos regularmente e $58 \%$ reduziram o número de parceiros sexuais. Parker chama a atenção para a magnitude destes achados, embora ressalte o cuidado que se deve ter na generalização destes resultados para homens que mantêm relações homossexuais. É preciso não perder de vista a diversidade sócio-cultural da subcultura homossexual no Brasil (Parker, 1990).

Deste modo, o conhecimento dos meios de transmissão do vírus parece ter evoluído, na medida em que os entrevistados, ao apontarem as formas de transmissão sexual, não se restringem às relações homossexuais masculinas. No entanto, se os meios de comunicação foram eficientes para romper com o conceito da SIDA/AIDS como "doença gay", o mesmo êxito não foi obtido, ao que parece, em relação a outros aspectos da transmissão da doença, e menos ainda quanto a mudanças de comportamento.

Embora mais de $90 \%$ dos nosses entrevistados reconheçam o sangue enquanto um veículo de transmissão do vírus, tanto no que se refere ao uso de drogas injetáveis quanto à transfusão, não há, contudo, uma discrimininação entre o ato de doar e o de receber a transfusão. É possível que informações contraditórias veiculadas em meados da década de 80 , como a transmissão do VIH através do beijo na boca e da picada de mosquito, tenham alguma influência sobre as respostas obtidas. A noção do mosquito enquanto transmissor da SIDA/AIDS talvez também esteja associada a campanhas dirigidas à prevenção de outras doenças, já que este vetor tem sido historicamente mencionado, nas políticas oficiais de educação em saúde, como fonte geradora de diversas patologias. No momento em que foi feita a pesquisa, o seu papel era enfatizado pelos meios de comunicação devido à epidemia de dengue.

Estas questōes, somadas ao relativo descrédito no preservativo enquanto meio eficaz de prevenção (Figura 2), podem contribuir para a compreensão dos fatores que levam à não-adoção de medidas de proteção individual contra a transmissão sexual da doença. Parker (s/d) acredita que as campanhas, sobretudo as veiculadas pela televisão, tenham desempenhado um papel importante na difusão do conhecimento sobre a epidemia com relação a aspectos da doença e de suas formas de transmissão. Entretanto, o impacto das campanhas dirigidas ao público geral sobre as mudanças nos comportamentos de risco é pequeno. Esse autor afirma que as atividades desenvolvidas com grupos específicos, em nível local, são mais efetivas. Enquanto as atividades orientadas para a população geral seriam mais adequadas para a manutenção das mudanças ao longo do tempo, as atividades de promoção de saúde altamente específicas e dirigidas seriam mais efetivas para que tais mudanças de comportamentos de risco se concretizassem.

Em nossa opinião, dois tipos de campanha têm sido veiculados pela televisão. Um deles parece ter como proposição a difusão de informações sobre a doença e suas formas de transmissão, enquanto o outro objetiva principalmente as mudanças de comportamento. Se o primeiro tipo de campanha vem alcançando seu propósíto, o mesmo não pode ser dito do segundo. É possível que isto se deva à estratégia adotada, que visa despertar o medo como motivação para mudanças de comportamento, na medida em que o temor exagerado pode provocar uma reação de paralisia e impotência frente ao 
problema, determinando uma postura fatalista diante do risco de contrair o vírus.

Para um entendimento mais aprofundado deste comportamento, seriam necessárias outras linhas de investigacão que contemplassem estes e outros aspectos. A produção de um vídeo informativo realizado pelo Posto de Saúde após a análise dos dados da pesquisa indicou, nos depoimentos colhidos, um forte sentimento de resignação, associado à religiosidade, em relação à possibilidade de contaminação pelo vírus da SIDA/AIDS.

Por fim, não se pode deixar de considerar a importância do aspecto econômico como fator limitante ao uso regular do preservativo na população estudada. No momento da pesquisa, o preço de uma caixa com três unidades, do tipo mais simples (não-lubrificado), equivalia a 1,7\% do salário mínimo ou a 2.36 litros de leite tipo C. Portanto, ainda que o fator econômico não deva ser considerado o único empecilho à utilização do preservativo, não se pode deixar de assinalar o seu caráter restritivo.

\section{RESUMO}

FERNANDES, J. C. L.; COUTINHO, E. da S. F. \& MATIDA, A. Conhecimentos e Atitudes Relativas a SIDA/AIDS em uma População de Favela do Rio de Janeiro. Cad. Saúde Públ., Rio de Janeiro, 8 (2): 176 182, abr/jun, 1992.

Com o objetivo de avaliar o grau de conhecimento e atitudes relativos à SIDA/AIDS, realizou-se um inquérito numa amostra de moradores da Favela da Rocinha, Rio de Janeiro, com idades entre 13 e 45 anos. Optou-se pela metodologia denominada Rapid Survey Methodology, sobretudo pelo seu baixo custo e por permitir um conhecimento quase que imediato das questōes de maior interesse.

Embora a maior parte dos individuos da amostra tenha demonstrado um grau elevado de conhecimento no que se refere às formas de transmissão sexual do VIH, mais da metade destes referiu a picada de mosquito, a doação de sangue e o beijo na boca como meio de adquirir a SIDA/AIDS. Com relação ao uso de preservativos, apenas $57,6 \%$ dos entrevistados acreditaram tratar-se de um método seguro de prevenção, e somente $21,9 \%$ referiram utilizá-lo, ainda que de modo irregular.

Palavras-Chave: AIDS; Transmissão VIH; Conhecimento; Comportamentos; Prevenção

\section{REFERÊNCIAS BIBLIOGRÁFICAS}

FRERICHS, R. \& TAR TAR, K., 1989. Computer-Assisted Rapid Surveys in Developing Countries. Public Health Reports, 104: 14-23.

HENDERSON, R. H. \& SUNDARESAN, T., 1982. Cluster sampling to assess immunization coverage: a review of experience with a simplified sampling method. Bulletin of the World Health Organization, 60: 253-260.

MAY, S. B., 1991. Comunicação pessoal

MUNHOZ, R.; GRANJEIRO, A. \& SILVA, J. L., 1989. Pesquisa Vocé e a AIDS. São Paulo: Programa de Controle e Prevenção Contra a AIDS, Setor de Vigilância Epidemiológica do Centro de Referência e Treinamento AIDS-SP.

PARKER, R., 1990. Depois da AIDS: mudanças no comportamento (homo)sexual. In: AIDS. $A$ Terceira Epidemia (H. Daniel \& R. Parker), São Paulo: Iglu Editora.

, s/d. AIDS Education and Health Promotion in Brazil: lessons from the past and prospects for the future. In: AIDS Prevention Through Education: a world view (J. Mann; H. Fineberg \& J. Sepulveda), Oxford: Oxford University Press. (no prelo)

SES (Secretaria de Estado de Saúde - SUS/RJ), 1990. Boletim Epidemiológico sobre AIDS. Novembro/dezembro. Rio de Janeiro: SES. 